\title{
On the Differential Geometry of 3D Flow Patterns: Generalized Helicoids and Diffusion MRI Analysis
}

\author{
Peter Savadjiev \\ School of Computer Science \\ McGill University \\ Montréal, QC, Canada \\ petersvecim.mcgill.ca
}

\author{
Steven W. Zucker \\ Department of Computer Science \\ Yale University \\ New Haven, CT, USA \\ steven.zucker@yale.edu
}

\author{
Kaleem Siddiqi \\ School of Computer Science \\ McGill University \\ Montréal, QC, Canada \\ siddiqi@cim.mcgill.ca
}

\begin{abstract}
Configurations of dense locally parallel $3 D$ curves occur in medical imaging, computer vision and graphics. Examples include white matter fibre tracts, textures, fur and hair. We develop a differential geometric characterization of such structures by considering the local behaviour of the associated $3 D$ frame field, leading to the associated tangential, normal and bi-normal curvature functions. Using results from the theory of generalized minimal surfaces we adopt a generalized helicoid model as an osculating object and develop the connection between its parameters and these curvature functions. These developments allow for the construction of parametrized $3 D$ vector fields (sampled osculating objects) to locally approximate these patterns. We apply these results to the analysis of diffusion MRI data via a type of $3 D$ streamline flow. Experimental results on data from a human brain demonstrate the advantages of incorporating the full differential geometry.
\end{abstract}

\section{Introduction}

Configurations of dense locally parallel 3D curves arise in medical imaging, computer vision and graphics. Examples include white matter fiber tracts in diffusion MRI [3], texture patterns on surfaces [13], hair patterns [6], and 3D streamlines [26]. Whereas the sense of a type of flow induced by such structures is immediate (see Fig. 1), capturing the underlying geometry has proved difficult. Strikingly, the wide variety of methods proposed in the literature share the common theme of following only the streamline (tangent) direction. There is in fact rich geometric structure in the normal and bi-normal directions as well. For example, a fiber population in diffusion MRI, though approximately parallel locally, can diverge or branch and distinct fiber populations can cross (see Fig. 1). In this article we develop a differential geometric characterization of such structures by considering the local behaviour of the associated 3D frame field. We begin with a brief overview of existing modeling techniques in diffusion MRI, which is the application domain of this paper.

Diffusion MRI exploits the property that fibrous structures restrict the random thermal motion of water molecules in live tissue so that it tends to be maximal along their orientation [16]. A variety of representations can be used to model the diffusion at a given location. While the early methods impose a 3D Gaussian distribution model, represented as a diffusion tensor (DT) [3], model-free representations have now become popular, e.g. Q-ball (QB) reconstruction [24]. These latter high angular resolution methods represent the local diffusion profile as a sampled function on a sphere and allow for a large number of fibre orientations to be reflected at a single location.

The literature has incorporated geometry at different processing stages in order to accomplish the task of modeling white matter fibre tracts from diffusion MRI data. For example, differential geometry has been used to characterize the shape of individual fibres [4], to cluster together individual fibres [10], or to compute tensor statistics along fibres [8]. These techniques typically apply geometrical analysis on fibre paths that have been obtained in a preprocessing stage through a fibre tracking algorithm (see [17] for a review). Other studies analyze the geometry of diffusion tensors prior to extracting fibres through tracking algorithms, by treating diffusion tensors as elements in a Riemannian space of $3 \times 3$ symmetric positive-definite matrices. Some approaches, e.g. [15, 25], use metrics in such spaces to segment statistically similar groups of tensors. Others, e.g. [2], design metrics to approximate and interpolate tensor data using B-splines. These types of approaches have been used with some success as a preprocessing step for the segmentation of fibre populations. However, statistical approaches can have difficulty with cases of tensor fields that present similar statistics but belong to different fibre populations, or 

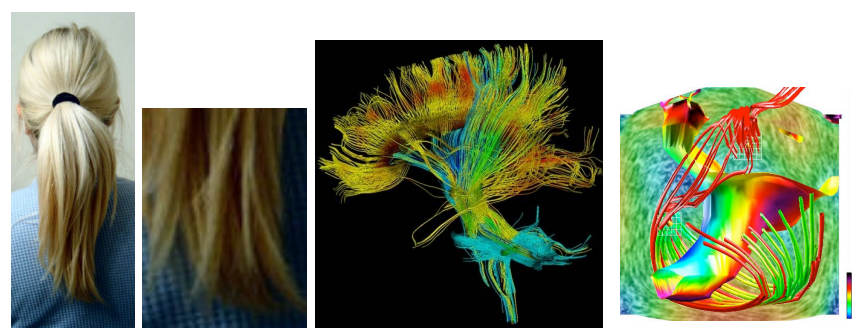

Figure 1. Examples of dense, locally parallel 3D curve flow patterns. Left: Human hair, with a zoom-in on the bottom of the pony-tail (source: www. search.com). Middle: a rendering in artificial colour of major white matter pathways in the human brain (source: www.medical.siemens.com). Right: Visualization of tumble motion using 3D streamlines and a combination of other techniques [14] (reproduced with permission from the author).

tensor fields that locally have different statistics but belong in fact to the same populations (e.g. in crossing regions).

All of the above techniques, as well as the modeling of hair and fur patterns in computer vision and graphics, could benefit from an analysis of the 3D streamline flow geometry of fibers, which is the main technical contribution of this article. We develop a differential geometric model of 3D streamline flow in Section 2 and introduce a notion of flow compatibility between nearby orientation measurements, motivated by the literature on minimal surfaces. We apply these results to the analysis of diffusion MRI in Section 3 and conclude with a discussion in Section 4.

\section{3D Streamline Flow}

We refer to a configuration of dense, locally parallel curves or streamlines in three dimensional Euclidean space $\mathbf{E}^{3}$ as a 3D streamline flow ${ }^{1}$. Such a flow can bend in the directions tangent, normal and bi-normal to each streamline. In order to model this behaviour, one needs to introduce an appropriate representation of the flow. At each point along each streamline we consider a local frame defined by the unit tangent, normal and bi-normal vectors. The set of all these frames is referred to as a "frame field" [19]. The use of a frame field allows for a natural representation of the geometric properties of the flow, since it is a generalization of the concept of the Frenet frame used to describe the geometry of individual curves. In the following section we develop the differential geometry of the frame field.

\footnotetext{
${ }^{1}$ We note that this construct is distinct from the idea of a flow texture in graphics, used for fluid flow visualization.
}

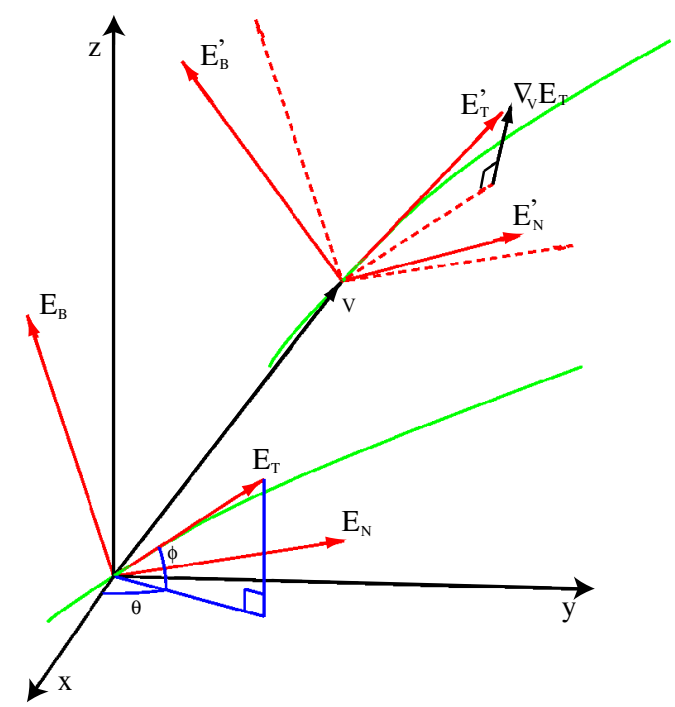

Figure 2. A 3D streamline flow is represented as a frame field, locally tangent to the flow streamlines (green). The tangential component $E_{T}$ of the frame is defined by orientation functions $\theta$ and $\phi$ with respect to a global coordinate system. A small translation of frame $\left(E_{T}, E_{N}, E_{B}\right)$ along vector $V$ results in a new frame $\left(E_{T}^{\prime}, E_{N}^{\prime}, E_{B}^{\prime}\right)$, rotated with respect to the original frame by an angle determined by the covariant derivative of $E_{T}$ in direction $V$, $\nabla_{V} E_{T}$. Expressing this covariant derivative in terms of the frame itself yields the three curvatures $\kappa_{T}, \kappa_{N}, \kappa_{B}$.

\subsection{Differential Geometry}

Consider a frame field given in spherical coordinates in Euclidean 3D space $\mathbf{E}^{3}$ :

$$
\begin{aligned}
& E_{T}=(\cos \phi \cos \theta, \cos \phi \sin \theta, \sin \phi) \\
& E_{N}=(-\sin \theta, \cos \theta, 0) \\
& E_{B}=(-\sin \phi \cos \theta,-\sin \phi \sin \theta, \cos \phi)
\end{aligned}
$$

Here $\theta$ and $\phi$ are two orientation functions that describe the orientation of vector $E_{T}$ with respect to a fixed global coordinate system. Angle $\phi$ represents elevation from the $x y$ plane, and $\theta$ represents the angle between the $x$-axis and the orthographic projection of $E_{T}$ along the $z$-axis onto the $x y$ plane.

Fig. 2 illustrates an example of a $\left(E_{T}, E_{N}, E_{B}\right)$ frame located (without loss of generality) at the origin of the global coordinate system. We are interested in characterizing the differential behaviour of the frame as it moves within a local volume. In particular, we seek a representation of this behaviour in terms of the frame itself. We begin by expressing the covariant derivatives of the frame vectors. Using Theorems 7.2 and 7.3 in Ch. 2 of [19], they are given by:

$$
\left[\begin{array}{c}
\nabla_{V} E_{T} \\
\nabla_{V} E_{N} \\
\nabla_{V} E_{B}
\end{array}\right]=\Omega\left[\begin{array}{c}
E_{T} \\
E_{N} \\
E_{B}
\end{array}\right],
$$


where

$\Omega=\left[\begin{array}{ccc}0 & \cos (\phi) d \theta(V) & d \phi(V) \\ -\cos (\phi) d \theta(V) & 0 & \sin (\phi) d \theta(V) \\ -d \phi(V) & -\sin (\phi) d \theta(V) & 0\end{array}\right]$.

Here $\nabla_{V} E$ represents the covariant derivative of vector $E$ in the (arbitrary) direction $V$, and $d \phi(V)$ and $d \theta(V)$ are 1-forms such that $d \phi(V)=\nabla \phi \cdot V$ and $d \theta(V)=\nabla \theta \cdot V$.

The $\left(E_{T}, E_{N}, E_{B}\right)$ frame is a rigid object, and so its local behaviour is captured entirely by the covariant derivative $\nabla_{V} E_{T}$. Since covariant derivation is a linear operation,

$$
\begin{aligned}
\nabla_{V} E_{T} & =\nabla_{a V_{1}+b V_{2}+c V_{3}} E_{T} \\
& =a \nabla_{V_{1}} E_{T}+b \nabla_{V_{2}} E_{T}+c \nabla_{V_{3}} E_{T},
\end{aligned}
$$

where $a, b$ and $c$ are constants, and $V_{1}, V_{2}$ and $V_{3}$ are any set of three linearly independent vectors such that $V=$ $a V_{1}+b V_{2}+c V_{3}$. A natural choice for a basis of three linearly independent vectors are the frame vectors themselves. Thus, to express $\nabla_{V} E_{T}$ in terms of the frame itself, we write

$$
\begin{aligned}
\nabla_{V} E_{T} & =\nabla_{a E_{T}+b E_{N}+c E_{B}} E_{T} \\
& =a \nabla_{E_{T}} E_{T}+b \nabla_{E_{N}} E_{T}+c \nabla_{E_{B}} E_{T} .
\end{aligned}
$$

In other words, $\nabla_{V} E_{T}$ is expressed as a sum of three vectors, each of which describes the covariant derivative of $E_{T}$ in the directions of $E_{T}, E_{N}$ and $E_{B}$, respectively.

This leads to the definition of the magnitude of the tangential, normal and bi-normal curvature functions: ${ }^{2}$

$$
\begin{aligned}
\left\|\kappa_{T}\right\| & =\left\|\nabla_{E_{T}} E_{T}\right\|=\left\|\cos \phi d \theta\left(E_{T}\right) E_{N}+d \phi\left(E_{T}\right) E_{B}\right\| \\
\left\|\kappa_{N}\right\| & =\left\|\nabla_{E_{N}} E_{T}\right\|=\left\|\cos \phi d \theta\left(E_{N}\right) E_{N}+d \phi\left(E_{N}\right) E_{B}\right\| \\
\left\|\kappa_{B}\right\| & =\left\|\nabla_{E_{B}} E_{T}\right\|=\left\|\cos \phi d \theta\left(E_{B}\right) E_{N}+d \phi\left(E_{B}\right) E_{B}\right\| .
\end{aligned}
$$

These curvature functions describe the differential behaviour of $E_{T}$, and thus the $\left(E_{T}, E_{N}, E_{B}\right)$ frame as it moves within a local neighbourhood. Fig. 2 provides the intuition of how a small translation of the frame $\left(E_{T}, E_{N}, E_{B}\right)$ along vector $V$ results in a rotation determined by $\nabla_{V} E_{T}$.

The differential geometry developed thus far applies to the analysis of 3D streamline flow curves that have been provided as input. In practice, however, we are faced with a type of "chicken-and-egg" problem. With input data in the form of local orientation measurements the underlying 3D streamline flow must first be inferred. We approach this problem by developing a notion of transport along a 3D streamline flow. The key technical contribution is to adopt an appropriate osculating object model and to relate its parameters to the curvature functions developed above.

\footnotetext{
${ }^{2}$ Adding a sign to each specifies the direction of bending with respect to the frame field at the origin.
}

\subsection{Transport}

For planar curves in 2D Euclidean space $\mathbf{E}^{2}$, the Euclidean curvature $\kappa$ at a point provides the osculating disc by which a tangent measurement can be transported to obtain nearby tangents on the curve, as exploited in [20]. For non-planar curves in $\mathbf{E}^{3}$, a notion of transport is provided by an osculating helix, as used in [21] for 3D curve inference in diffusion MRI. The more general case of 2D streamline (or texture) flow has been addressed in [5], where a right helicoid model has been used as an osculating object. Extending these ideas to the case of 3D streamline flow is non-trivial and a key contribution of this paper is the use of minimal surface theory to resolve this question. To illustrate some of the complexity, the $\left(E_{T}, E_{N}, E_{B}\right)$ frame field is described by two orientation functions, $\theta$ and $\phi$, defined over a volume in $\mathbf{E}^{3}$. Thus, a dual representation of the $\left(E_{T}, E_{N}, E_{B}\right)$ frame field is that of two hypersurfaces in $\mathbf{E}^{4}: \theta(x, y, z)$ and $\phi(x, y, z)$. We seek a "minimal" osculating object model, i.e., one that determines these hypersurfaces and which has three degrees of freedom, each related to one of the curvature functions of Eq. (8).

Without loss of generality, we assume that the point along the 3D streamline flow for which an osculating object model is sought is located at the origin. Results from the theory of minimal surfaces combined with the differential geometry of 3D streamline flow leads to the following propositions.

Proposition 1 A generalized (or "stacked") helicoid model for $\theta$, given by

$$
\theta(x, y, z)=\tan ^{-1}\left(\frac{K_{T} x+K_{N} y}{1+K_{N} x-K_{T} y}\right)+K_{B} z,
$$

with $K_{T}, K_{N}, K_{B}$ as constants, is a minimal hypersurface.

Proposition 2 Equating $\phi$ with $\theta$ leads to the following relationship between the constants $K_{T}, K_{N}, K_{B}$ of the stacked helicoid and the curvatures (via Eq. (8)) of a unique $3 D$ streamline flow:

$$
\begin{aligned}
& \kappa_{T}(0,0,0)=\sqrt{2}\left\|K_{T}\right\| \\
& \kappa_{N}(0,0,0)=\sqrt{2}\left\|K_{N}\right\| \\
& \kappa_{B}(0,0,0)=\sqrt{2}\left\|K_{B}\right\| .
\end{aligned}
$$

Osculating Object Using these propositions, an osculating object for 3D streamline flow is obtained by a frame field defined by Eqs.(1)-(3), with orientation functions $\theta=$ $\phi$ specified by Eq. (9).

Proof of Proposition 2 Eq. (10) can be obtained by computing the gradient of $\theta(x, y, z)$ in Eq. (9) and noting that at the origin, $\nabla \theta(0,0,0)=\left(K_{T}, K_{N}, K_{B}\right)$. Since 
$\theta(0,0,0)=0=\phi(0,0,0)$, this aligns the $\left(E_{T}, E_{N}, E_{B}\right)$ frame at the origin with the global $(x, y, z)$ coordinate system, so that $E_{T}=(1,0,0), E_{N}=(0,1,0)$ and $E_{B}=$ $(0,0,1)$. Eq. (10) follows from substituting the values for $E_{T}, E_{N}, E_{B}, \nabla \theta$ and $\nabla \phi$ into the curvature functions of Eq. (8).

Sketch of Proof of Proposition 1 In order to show that the model for $\theta$ presented in (9) is a minimal hypersurface in $\mathbf{E}^{4}$, consider that the term $\tan ^{-1}\left(\frac{K_{T} x+K_{N} y}{1+K_{N} x-K_{T} y}\right)$ represents a right helicoidal surface over the $x y$ plane in 3D Euclidean space [5], which is known by the classical theorem of Catalan to be a minimal surface in $\mathbf{E}^{3}$ (see e.g. [1]). The addition of the $K_{B} z$ term in (9) adds a linear coordinate function along the $z$ axis, so that $\theta(x, y, z)$ is a generalized helicoid hypersurface in $\mathbf{E}^{4}$ that can be expressed with a parametric equation

$$
\left(x_{1}, x_{2}, x_{3}, x_{4}\right)=\left(u \cos \left(t_{1} s\right), u \sin \left(t_{1} s\right), t_{2} s, v\right),
$$

where $x_{1}, x_{2}, x_{3}, x_{4}$ are coordinate functions, $u, v, s$ are parameters $\in R$, and $t_{1}$ and $t_{2}$ are real constants.

The concept of generalized helicoids in $\mathbf{E}^{n}$ space has been studied in [7, 22, 1, 9]. Blair and Vanstone show in [7] that complete ruled minimal $n$-dimensional hypersurfaces $M^{n}$ in $\mathbf{E}^{n+1}$ are products of $\mathbf{E}^{n-2}$ and a helicoid in $\mathbf{E}^{3}$. In particular, in $\mathbf{E}^{4}$, a generalized helicoid is the product of a helicoid in $\mathbf{E}^{3}$ and the real line. Barbosa et al. [1] and Thas [22] independently derive the parametric equations of such minimal generalized helicoids (see also [23]), which in the case of $\mathbf{E}^{4}$ work out to be Eq. (11) [1, 22, 23]. The first, second and third parametric equations together represent a helicoid in $\mathbf{E}^{3}$, and the fourth parametric equation is a linear coordinate function.

Remark 1 There is an apparent relation of this result to the work of Hayden [11] on generalized helical curves. Hayden proves that in Riemannian spaces of $n$ dimensions, where $n$ is even, there does not exist a non-degenerate generalized helix, i.e., at least one of its curvatures must vanish. One can deduce from this result that in $\mathbf{E}^{4}$, a generalized helix can have non-null curvature and torsion, but higherorder curvatures must be null. Thus, in $\mathbf{E}^{4}$, a generalized helix must be the ordinary $3 \mathrm{D}$ helix with a linear coordinate function describing its fourth dimension.

Remark 2 The differential geometry of streamline flows in 2D has been developed in [5]. Motivated by energy minimization considerations, and the desire to maintain a constant ratio of tangential vs. normal curvature, the right helicoid

$$
\theta(x, y)=\tan ^{-1}\left(\frac{K_{T} x+K_{N} y}{1+K_{N} x-K_{T} y}\right)
$$

is used as an osculating object. Here, the osculating object $\theta$ is a surface in $\mathbf{E}^{3}$, and $K_{T}$ and $K_{N}$ represent the values of the tangential and normal curvatures, respectively, at the origin. It turns out that the curvature functions used in [5] are a special case of the more general curvatures defined by Eq. (8). Observe that in the case where $\phi=0$ everywhere, $E_{T}$ lies in the $x y$ plane, the problem is reduced to a two-dimensional one and Eq. (8) reduces to $\kappa_{T}=d \theta\left(E_{T}\right)$, $\kappa_{N}=d \theta\left(E_{N}\right)$ ( $\kappa_{B}$ vanishes). These are precisely the definitions of the tangential and normal curvatures proposed in [5] for 2D streamline flow analysis.

\section{Applications}

\subsection{Generalized Helicoids}

The first natural application of the above osculating object model is its use in generating various 3D streamline flows, each corresponding to a different set of curvature choices. We combine the two orientation functions $\theta$ and $\phi$ to represent spherical coordinates and to generate a vector field that corresponds to the tangential $E_{T}$ component of the frame field $\left(E_{T}, E_{N}, E_{B}\right)$. Fig. 3 visualizes the vector field $(x, y, z)=(\cos \phi \cos \theta, \cos \phi \sin \theta, \sin \phi)$, where $\theta$ and $\phi$ are defined as in Eq. (9), for three different combinations of values for $\left(K_{T}, K_{N}, K_{B}\right)$. The visualization shows three orthogonal slices passing through the origin, which is located at the central voxel of each neighbourhood. $E_{T}$ at the origin is set parallel to the $x$ axis. As expected, one observes vector fields with smoothly varying orientations but with rich geometry controlled by the curvature choices. In the top row, for which $K_{T}=0.2, K_{N}=K_{B}=0$, one observes no changes in the vectors in the $y z$ slice, as expected, since the $E_{N}$ and $E_{B}$ frame vectors at the origin (not shown) are aligned with the $y$ and $z$ axes, respectively. In the $x z$ slice, change is observed as the vector location moves along the $x$ axis but not along the $z$ axis. In the middle row, where $K_{N}$ is non-null, but $K_{B}=0$, one starts to observe a change in the vector direction as the location moves along the $y$ axis, associated with $E_{N}$ at the origin. Finally, when all of $\left(K_{T}, K_{N}, K_{B}\right)$ are non-null, one always observes changes in the vector directions as their location moves in space.

\subsection{Diffusion MRI Regularization}

\subsubsection{Methodology}

We now use our generalized helicoid osculating object to infer 3D flow patterns from incomplete 3D orientation data, i.e., diffusion MRI datasets. It is incomplete in the sense that the data is spatially discretized, i.e., it is defined on a 3D cubic lattice. The data also has a limited angular resolution which depends on acquisition parameters. Finally, the diffusion profile is related to the underlying fibre structure, 


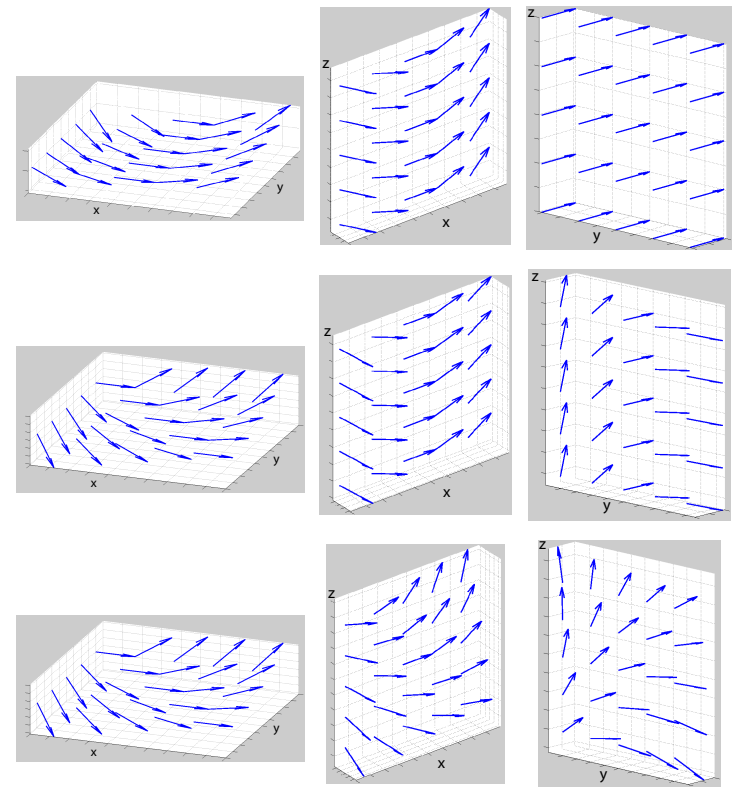

Figure 3. Three examples of a generalized helicoid osculating object for 3D streamline flow analysis, each visualized in three mutually orthogonal slices of a $5 \times 5 \times 5$ volume. Top row: $K_{T}=0.2$, $K_{N}=K_{B}=0$. Middle row: $K_{T}=K_{N}=0.2, K_{B}=0$. Bottom row: $K_{T}=K_{N}=K_{B}=0.2$.

but there can be also diffusion unrelated to fibre geometry, which can introduce ambiguities.

At each location (voxel) in the discretized 3D volume, the data is represented as an antipodally symmetric function on a sphere, called the diffusion orientation distribution function (ODF) [24]. In a manner similar to that of [21], the diffusion ODF is sampled along a fixed set of $m$ directions, providing a measure of confidence in the presence of a fibre oriented along each direction. An iterative relaxation labeling process $[12,5,21]$ is set up so that orientations that are supported by other nearby orientations via the helicoidal model gain confidence, while others are suppressed. Since a priori the parameters $\left(K_{T}, K_{N}, K_{B}\right)$ of the osculating object that describes best the flow pattern associated to orientation $\lambda$ at voxel $i$ are not known, we begin by generating a set of osculating objects, defined through Eq. (9), for a discretized range of values for $\left(K_{T}, K_{N}, K_{B}\right)$. These osculating objects, which are 3D vector fields like the ones illustrated in Fig. 3, are sampled at the centre of each voxel in a neighbourhood of a given size. Each such sampled vector field represents a particular combination of values for $\left(K_{T}, K_{N}, K_{B}\right)$ and is thus referred to as a 'curvature class'. Then, for a given orientation $\lambda$ at a given voxel $i$, for each curvature class, a support measure $s_{i}(\lambda)$ is calculated as

$$
s_{i}(\lambda)=\prod_{j} c \cdot p_{j}\left(\lambda^{\prime}\right),
$$

where index $j$ ranges over all voxels in the neighbourhood of $i$, and $\lambda^{\prime}$ is the index of the direction among the $m$ sampling directions that is closest to the osculating object vector at location $j$ (each voxel $j$ may have a different $\lambda^{\prime}$ ). $p_{j}\left(\lambda^{\prime}\right)$ denotes the value of the ODF at voxel $j$ sampled along its direction $\lambda^{\prime}$, and $c$ is a normalizing scalar constant. This (local) support function is then incorporated in a global measure of average local support

$$
A=\sum_{i=1}^{n} \sum_{\lambda=1}^{m} p_{i}(\lambda) s_{i}(\lambda)
$$

which is defined over the entire volume and is maximized using an iterative relaxation labeling algorithm ${ }^{3}$ [12]. Theoretical considerations [12] show that maximizing $A$ is equivalent to solving a system of variational equalities and has the effect of regularizing the data. At each iteration, the sampled ODF values $p_{i}(\lambda)$ are updated (see Algorithm 8.2 in [12]) as a function of the support $s_{i}(\lambda)$ they receive via the flow model, and this leads to an overall increase of the value of $A$. Since the iterative process is an any-time algorithm, it is not necessary in practice to attain a maximum of $A$, and it can thus be stopped at a desired level of regularization.

\subsubsection{Experimental Results}

Fig. 4 shows the result of applying the above 3D streamline flow analysis to diffusion MRI data from a human brain. It consists of two co-registered datasets, one with DT data and one with QB ODFs, and was obtained as described in [21].

The top row in Fig. 4 shows a region of interest (ROI) delimited with a white rectangle in the anatomical and the RGB image of the data, with the corresponding unregularized DT and QB data. The RGB image encodes the principal diffusion direction of the DT dataset, so that in this view, green denotes left-right orientation, blue denotes up-down orientation, and red denotes orientation out of the page. In this ROI one observes a crossing between the cortico-spinal tract (CST, blue), which fans out to the cortex and the superior longitudinal fasciculus (SLF, green). For clarity, both the unregularized DT and the unregularized QB datasets are visualized after subtraction of the minimal inscribed sphere. One can immediately see that the DT dataset, due to its representational limitations, cannot capture the intricate structure of the fibres that the QB dataset shows evidence for.

The bottom row shows the output of the 3D curve inference algorithm of [21] run on the DT dataset, followed by the output of our 3D streamline flow regularization algorithm run on the DT and QB datasets. To generate the latter two results, our 3D flow regularization algorithm used osculating objects that were sampled in a $3 \times 3 \times 3$ neighbourhood, with values for $K_{T}, K_{N}, K_{B}$ ranging from -0.1 to

\footnotetext{
${ }^{3}$ Although we adopt a relaxation labeling framework, quadratic programming and belief propagation algorithms could be used in its place.
} 

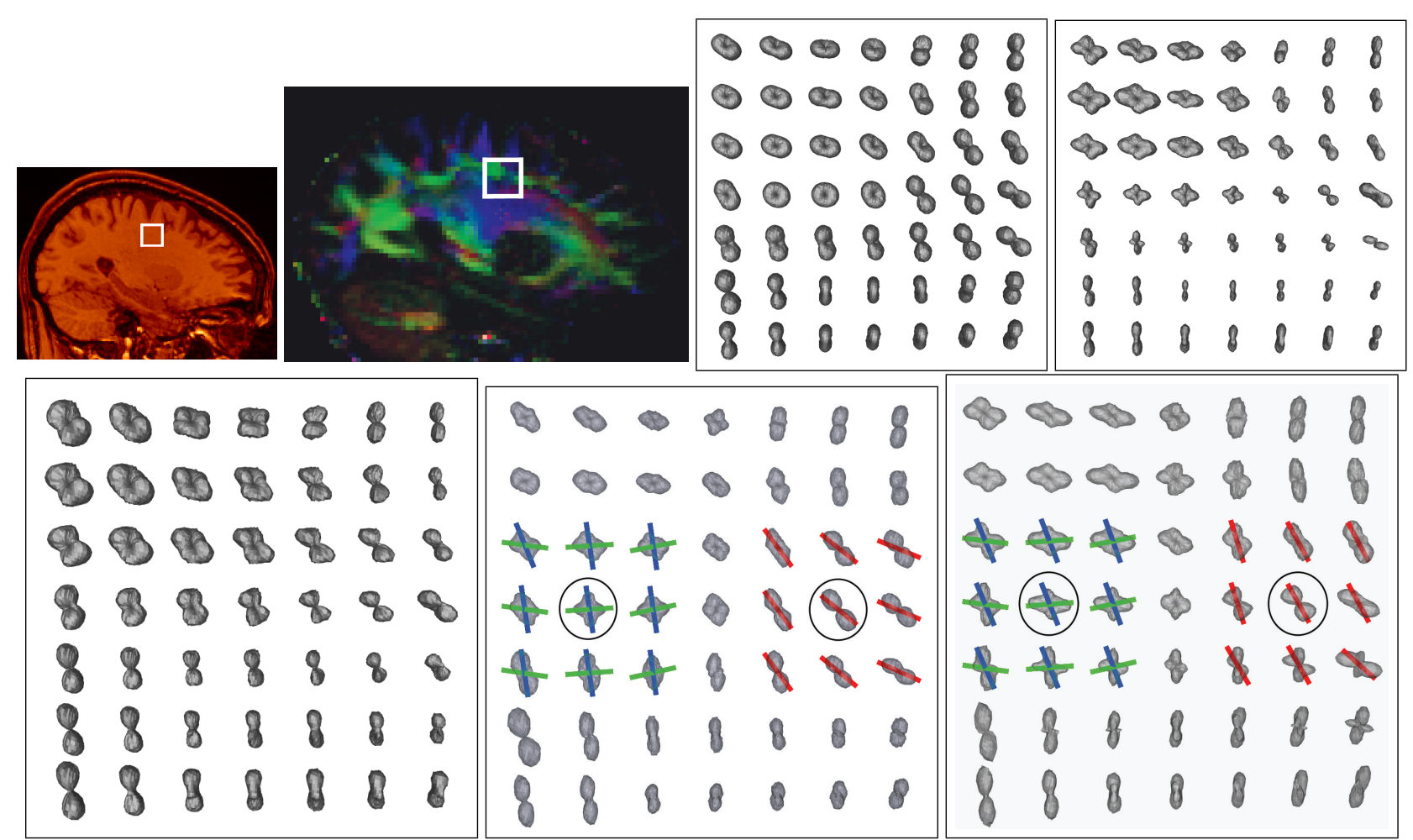

Figure 4. Inference of 3D flows in diffusion MRI data. Top row, from left to right: an ROI delimited by a white rectangle in the anatomical image (1) and the RGB image (2), followed by the unregularized DT dataset (3) and the unregularized QB dataset (4). Bottom row, from left to right: regularization results in this ROI, obtained by running 3D curve inference [21] on the DT dataset (1), and by 3D streamline flow inference on the DT dataset (2) and on the QB dataset (3). See the text for further details.

0.1. These figures also show the osculating objects associated with the ODF maxima in the circled voxels. For each ODF maximum in the selected (circled) voxel, we visualize those elements of the $3 \times 3 \times 3$ osculating object that are located within the slice of the ROI. Since these are osculating objects computed for the circled voxels, they are not expected to precisely match the ODF maxima in neighbouring voxels.

Observe that 3D streamline flow regularization of the DT dataset infers ODFs with multiple maxima that are qualitatively very similar to those in the QB dataset. Thus, it better captures the underlying fibre structure than the unregularized DT dataset. Since the unregularized QB dataset contains a lot more structural information than the DT dataset, the difference with the QB regularization result is less apparent. Regularizing the QB dataset has the effect of sharpening the ODFs and enhancing certain maxima that were originally less obvious, e.g., the voxels in the bottom right part of the ROI. The output of the 3D curve inference algorithm [21] run on the same DT dataset does capture the fibre geometry, but has more difficulty recovering some of the crossings in that area. In part this can be explained by the fact that the 3D streamline flow gains stronger support when locally parallel curve configurations are present.
To provide a stronger sense in 3D of the inferred flows, Fig. 5 takes the blue, green and red osculating objects shown for the two circled ODFs in the DT regularization result (Fig. 4, second row, middle), supersamples and visualizes them, using the same colours, in a manner similar to that of Fig. 3. To obtain a consistent visualization, for each osculating object the tangential component of the flow at the origin is aligned with the $x$-axis, and the neighbouring components are visualized in three mutually orthogonal slices, each oriented along one of the three coordinate axes. The curvature parameters for these osculating objects work out to be $K_{T}=0.1, K_{N}=0.1, K_{B}=-0.1$ (red, top row); $K_{T}=0.1, K_{N}=0, K_{B}=0$ (green, middle row); $K_{T}=-0.1, K_{N}=0, K_{B}=0$ (blue, bottom row).

Observe how the red osculating object in Fig. 4 (bottom row, middle and right) approximates the ascending U-fibre, while the two others capture the crossing between the CST and the SLF. Such inferred flow patterns can potentially be developed and used in an algorithm that would segment different fiber pathways based on 3D streamline flow coherence. Although outside the scope of this paper, the problems of fibre segmentation and clustering are of current interest in diffusion MRI analysis (e.g., see [18]), and the osculating objects inferred by $3 \mathrm{D}$ streamline flow could play 


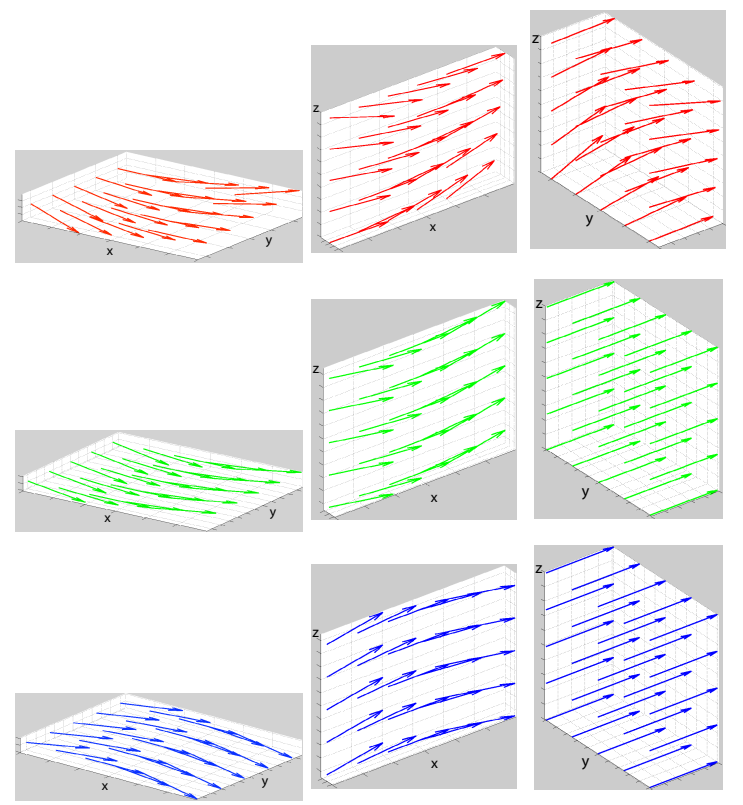

Figure 5. Each row shows one of the inferred flows for the circled voxels in Fig. 4 (bottom row, middle), supersampled in three mutually orthogonal slices through a $5 \times 5 \times 5$ neighbourhood, centered at the origin and aligned at the origin with the $x$ axis (for consistency in the visualization). The colours of the flow correspond to the colours in Fig. 4 (bottom row, middle).

an important role. Below we sketch a simple method based on 3D streamline flow for clustering.

The idea is to group ODF maxima together based on orientation and osculating object coherence. Two neighbouring ODF maxima are considered part of the same cluster, or fibre tract, if they belong to a similar curvature class, and if they are well aligned with one another's osculating objects. A measure of alignment between an ODF maximum $m^{\prime}$ defined at voxel $i$, and the osculating object of a neighbouring ODF maximum $m^{\prime \prime}$ defined at location $j$ is given by the angle between $m^{\prime}$ and the sample of the osculating object associated with $m^{\prime \prime}$ that corresponds to location $i$.

As an illustrative example, one can start with the leftmost circled voxel in Fig. 4 (bottom row, middle), and for each ODF maximum recursively group together neighbouring ODF maxima that obey the criteria of similarity in curvature classes and similarity in orientation with the underlying osculating objects. Fig. 6 shows the resulting clustering of ODF maxima in two groups: in blue, those that are associated with the CST and in green, those that are associated with the SLF. A 10-slice cluster volume is shown (Fig. 6, top) projected on a planar set of ODFs in a coronal slice from the regularized DT dataset discussed above (in relation to Fig. 4). To give a stronger sense of the 3D nature of the cluster volumes, Fig. 6 (bottom right) shows a zoomin viewed from an angle. In both cases, the circled voxel

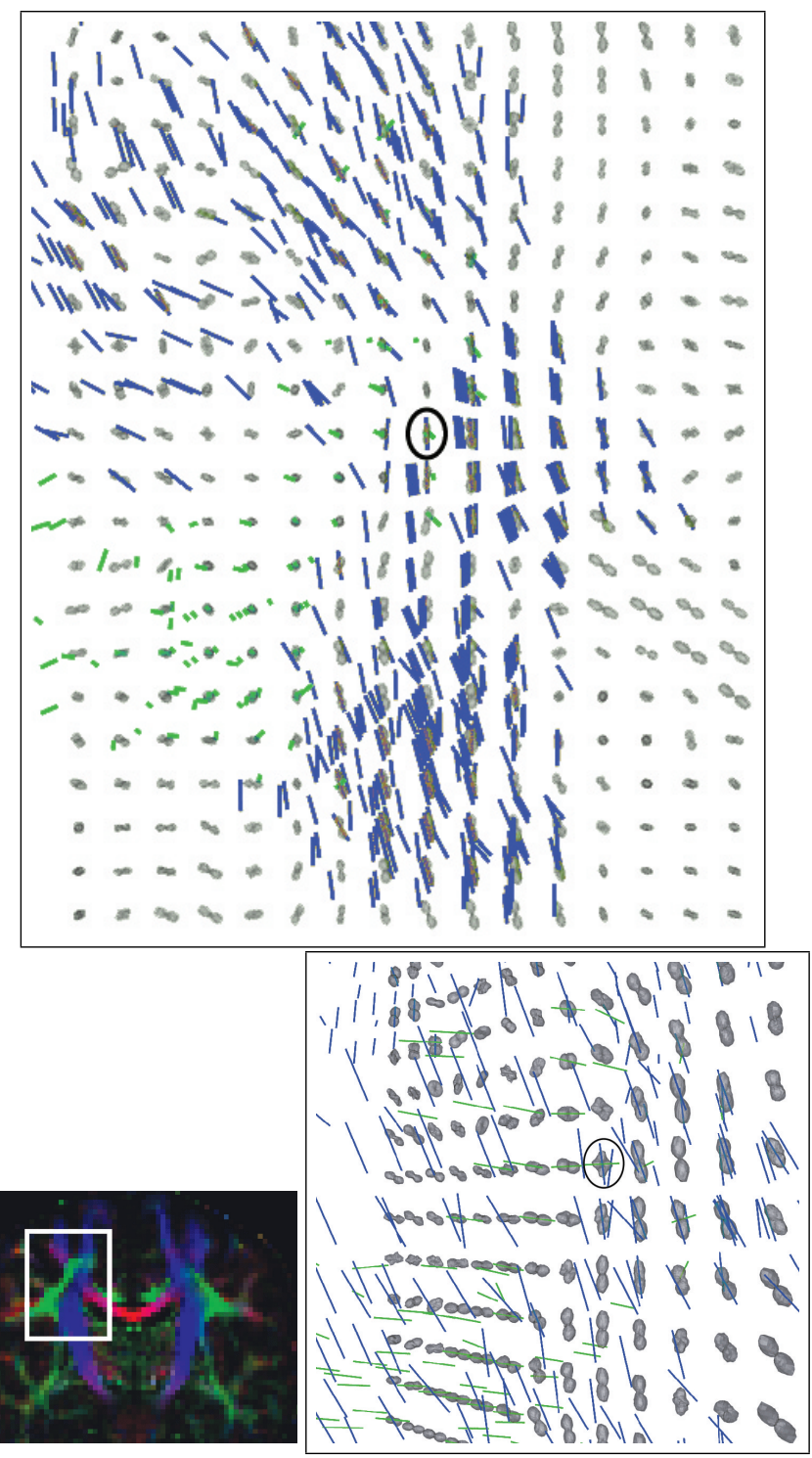

Figure 6. An illustration of ODF maxima clustering based on osculating object coherence. Starting from the leftmost circled voxel in Fig. 4 (bottom row, middle), neighbouring ODF maxima are locally clustered as belonging to the CST (blue) or the SLF (green). Top: a volume of clustered ODF maxima is projected onto a coronal slice through the regularized DT dataset (see text). The circled voxel is the same as in Fig. 4 (bottom row, middle). Bottom row: the slice through the ODF dataset is indicated with a white rectangle in the RGB image (left); a zoom-in on the circled voxel viewed from an angle is provided (right).

(and the colour coding of ODF maxima) is the same as the leftmost circled voxel in Fig. 4 (bottom row, middle). The set of ODFs viewed in Fig. 6 (top) is delimited with a white rectangle in the RGB image of the ODF dataset (Fig. 6, bottom left), in which blue denotes up-down orientation, and green denotes orientation out of the page. These clusters are $3 \mathrm{D}$ vector fields, and their projection on the image plane 
causes perspective effects. These are preliminary results, and though visually plausible (see the RGB image) claims of anatomical validity would be premature. Nonetheless, they demonstrate the usefulness of our differential geometric characterization of fiber tracts as a $3 \mathrm{D}$ streamline flow.

\section{Conclusion}

Fibre tracts, hair and fur patterns follow a 3D characterization of the Frenet geometry. We have developed the local structure for these patterns as a generalized helicoid and have demonstrated how it could be used in the context of diffusion MRI regularization for inferring the structure of crossing and curving fibre tracts, as well as the associated local 3D streamline flow patterns. We foresee a further application of the theory developed in this paper to the problems of fibre segmentation and clustering. Finally, applications to problems in computer vision, computer graphics and visualization exist as well. For example, articulated $2 \mathrm{D}$ patterns moving in space/time would follow similar geometry, which suggests a new foundation for use in object tracking.

Acknowledgments We are grateful to Jennifer S. W. Campbell and G. Bruce Pike for helpful discussions and for sharing the human brain data. This research was supported by grants from NSERC, FQRNT and PRECARN.

\section{References}

[1] J. M. Barbosa, M. Dajczer, and L. P. Jorge. Minimal ruled submanifolds in spaces of constant curvature. Indiana University Mathematics Journal, 33:531-547, 1984.

[2] A. Barmpoutis, B. C. Vemuri, and J. R. Forder. Robust tensor splines for approximation of diffusion tensor MRI data. In MMBIA'06, pages 86-93, 17-18 June 2006.

[3] P.-J. Basser, J. Matiello, and D. Le Bihan. MR diffusion tensor spectroscopy and imaging. Biophysical Journal, 66:259267, 1994.

[4] P. Batchelor, F. Calamante, J. Tournier, D. Atkinson, D. L. G. Hill, and A. Connelly. Quantification of the shape of fiber tracts. Magn. Res. in Medicine, 55:894-903, 2006.

[5] O. Ben-Shahar and S. W. Zucker. The perceptual organization of texture flow: A contextual inference approach. IEEE Trans. Pattern Analysis and Machine Intelligence, 25:401417, 2003.

[6] F. Bertails, B. Audoly, M.-P. Cani, B. Querleux, F. Leroy, and J.-L. Lévêque. Super-helices for predicting the dynamics of natural hair. In ACM Transactions on Graphics (Proceedings of SIGGRAPH), August 2006.

[7] D. E. Blair and J. R. Vanstone. A generalization of the helicoid. In Minimal Submanifolds and Geodesics, pages 13-16. Kaigai Publications, Tokyo, 1978.

[8] I. Corouge, P. T. Fletcher, S. Joshi, S. Gouttard, and G. Gerig. Fiber tract-oriented statistics for quantitative diffusion tensor
MRI analysis. Medical Image Analysis, 10(5):786-798, October 2006.

[9] F. Dillen. Ruled submanifolds of finite type. Proc. of the American Mathematical Society, 114:795-798, 1992.

[10] Z. Ding, J. C. Gore, and A. W. Anderson. Classification and quantification of neuronal fiber pathways using diffusion tensor MRI. Magn. Res. in Medicine, 49:716-721, 2003.

[11] H. A. Hayden. On a generalized helix in a Riemannian $n$ space. Proc. London Math. Soc. 2, 32:337-345, 1930.

[12] R. Hummel and S. W. Zucker. On the foundations of relaxation labeling processes. IEEE Trans. Pattern Analysis and Machine Intelligence, 5:267-287, 1983.

[13] D. C. Knill. Contour into texture: Information content of surface contours and texture flow. J. Opt. Soc. Am. A, 18(1):1235, 2001.

[14] R. S. Laramee, D. Weiskopf, J. Schneider, and H. Hauser. Investigating swirl and tumble flow with a comparison of visualization techniques. In VIS '04: Proceedings of the conference on Visualization '04, pages 51-58, Washington, DC, USA, 2004. IEEE Computer Society.

[15] C. Lenglet, M. Rousson, R. Deriche, and O. Faugeras. Statistics on the manifold of multivariate normal distributions: Theory and applications to diffusion tensor MRI processing. J. Mathematical Imaging and Vision, 25:423-444, 2006.

[16] C. P. Lin, V. Wedeen, J. Chen, C. Yao, and W. Tseng. Validation of diffusion spectrum magnetic resonance axonal fiber imaging with manganese-enhanced optic tracts and ex-vivo phantoms. NeuroImage, 19:482-495, 2003.

[17] S. Mori and P. C. M. van Zijl. Fiber tracking: principles and strategies - a technical review. NMR in Biomedicine, 15:468480, 2002.

[18] L. J. O’Donnell, M. Kubicki, M. E. Shenton, M. H. Dreusicke, W. E. L. Grimson, and C.-F. Westin. A method for clustering white matter fiber tracts. Am. J. Neuroradiol., 27:1032-1036, 2006.

[19] B. O'Neill. Elementary Differential Geometry. Academic Press, 1966.

[20] P. Parent and S. W. Zucker. Trace inference, curvature consistency, and curve detection. IEEE Trans. Pattern Analysis and Machine Intelligence, 11:823-839, 1989.

[21] P. Savadjiev, J. S. W. Campbell, G. B. Pike, and K. Siddiqi. 3D curve inference for diffusion MRI regularization and fibre tractography. Medical Image Analysis, 10(5):799-813, October 2006.

[22] C. Thas. Minimal generalized ruled surfaces in the Euclidean space $E^{m}$. Internal Report. Seminar of Higher Geometry, State University of Ghent, 1979.

[23] C. Thas. A property of the Veronese surface. Journal of Geometry, 32:157-168, 1988.

[24] D. Tuch. Q-ball imaging. Magn. Res. in Medicine, 52:1358$1372,2004$.

[25] Z. Wang and B. C. Vemuri. DTI segmentation using an information theoretic tensor dissimilarity measure. IEEE Trans. Medical Imaging, 24:1267-1277, 2005.

[26] S. Zhang, Ç. Demiralp, and D. Laidlaw. Visualizing diffusion tensor MR images using streamtubes and streamsurfaces. IEEE Trans. Visualization and Computer Graphics, 9:454-462, 2003. 